\title{
Theorists bridge space-time rips
}

Framework offers starting point to explaining how particles cope with fluctuations in gravity.

\section{BY EUGENIE SAMUEL REICH}

$\mathrm{C}$ ould an analysis based on relatively simple calculations point the way to reconciling the two most successful and stubbornly distinct - branches of modern theoretical physics? Frank Wilczek and his collaborators hope so.

The task of aligning quantum mechanics, which deals with the behaviour of fundamental particles, with Einstein's general theory of relativity, which describes gravity in terms of curved space-time, has proved an enormous challenge. One of the difficulties is that neither is adequate to describe what happens to particles when the space-time they occupy undergoes drastic changes - such as those thought to occur at the birth of a black hole. But in a paper posted to the arXiv preprint server on 15 October (A. D. Shapere et al. http://arxiv.org/ abs/1210.3545; 2012), three theoretical physicists present a straightforward way for quantum particles to move smoothly from one kind of 'topological space' to a very different one.

The analysis does not model gravity explicitly, and so is not an attempt to formulate a theory of 'quantum gravity' that brings general relativity and quantum mechanics under one umbrella. Instead, the authors, including Nobel laureate Frank Wilczek of the Massachusetts Institute of Technology (MIT) in Cambridge, suggest that their work might provide a simplified framework for understanding the effects of gravity on quantum particles, as well as describing other situations in which the spaces that quantum particles move in can radically alter, such as in condensed-matter-physics experiments. "I'm pretty excited," says Wilczek, "We have to see how far we can push it."

The idea is attracting attention not only because of the scope of its possible applications, but because it is based on undergraduate-level mathematics. "Their paper starts with the most elementary framework," says Brian Greene, a string theorist at Columbia
University in New York. "It's inspiring how far they can go with no fancy machinery."

Wilczek and his co-authors set up a hypothetical system with a single quantum particle moving along a wire that abruptly splits into two. The stripped-down scenario is effectively the one-dimensional version of an encounter

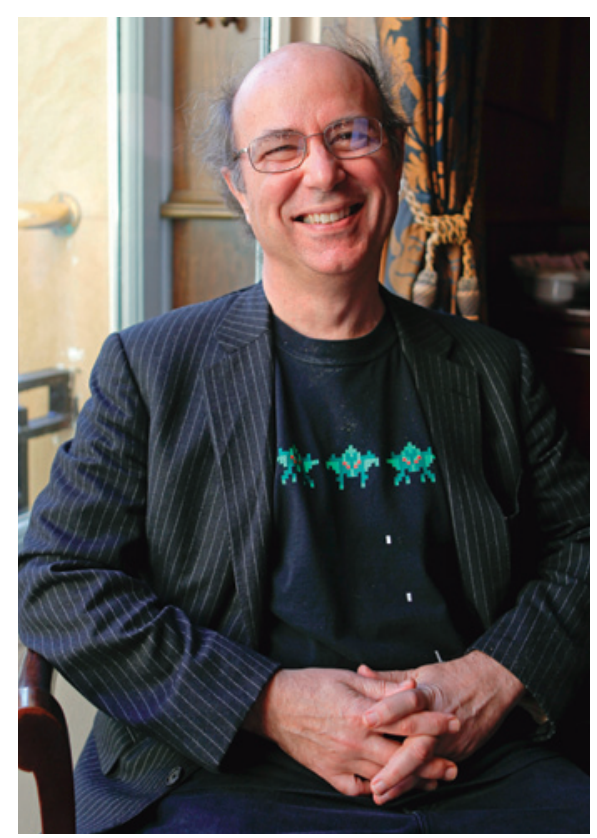

Frank Wilczek studies how fundamental particles respond to drastic changes in space-time.

with ripped space-time, which occurs when the topology of a space changes radically. The theorists concentrate on what happens at the endpoints of the wire - setting the "boundary conditions' for the before and after states of the quantum wave associated with the particle. They then show that the wave can evolve continuously without facing any disruptions as the boundary conditions shift from one geometry to the other, incompatible one. "You can smoothly follow this process," says
Al Shapere at the University of Kentucky in Lexington, a co-author on the paper, adding that, like a magician's rings, the transformation is impossible to visualize, but does make mathematical sense.

The desire to escape the mathematical headaches caused by such transformations is one motivation for string theory, which allows smooth changes in the topology of space-time, says Greene. He suggests that the approach developed by Wilczek, Shapere and MIT undergraduate student Zhaoxi Xiong could be applied within string theory too.

Although Wilczek originally believed that the result was new, a 1995 paper by Aiyalam Balachandran of Syracuse University in New York proposed a similar strategy for describing changes in topology in quantum mechanics (A. P. Balachandran et al. Nucl. Phys. B 446, 299-314; 1995). Balachandran acknowledges that his work hasn't hit the mainstream and says that he hopes Wilczek's paper will prompt others to take a closer look. "Conventional approaches to this problem don't get very far," he says. "This opens up a new technique."

The framework might also provide inspiration for experimentalists working on condensed matter. Rob Myers, a string theorist at the Perimeter Institute for Theoretical Physics in Waterloo, Canada, says that he expects it to be relevant to an area called quantum quenches, in which quantum systems evolve in isolation from the environment and are then kicked out of equilibrium by an action of the experimentalist. Condensed-matter physicists have developed several quantum systems including cold-atom traps and superconducting circuits - that can be used to test this idea.

Although the authors lay out their solution in only one dimension, Myers expects that the approach will readily generalize to describe real experiments in three dimensions. But he cautions that the paper represents only a first step. "To really see the impact of this work, that will take a while," he says. .
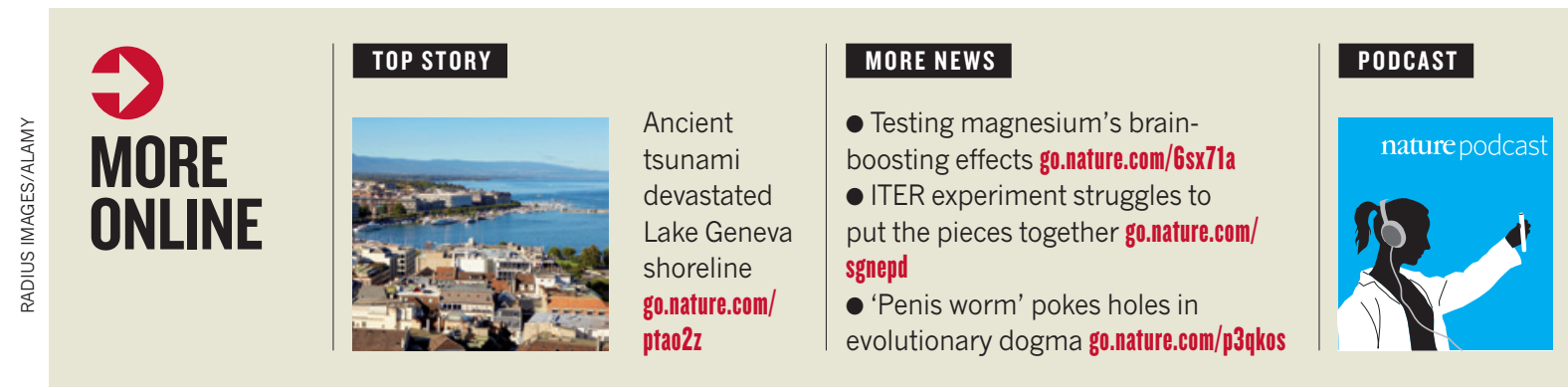

Cancer risk for redheads, mapping the bird family tree and single-cell sequencing go.nature.com/cykasc 